\title{
ON THE IMPLICIT COMPLEMENTARITY PROBLEM
}

\section{IN HILBERT SPACES}

\author{
G. ISAC
}

\begin{abstract}
We consider in this paper the implicit complementarity problem imposed by quasi-variational inequalities and stochastic optimal control. The principal result is an existence theorem for the implicit complementarity problem in Hilbert spaces.
\end{abstract}

1.

We consider in this paper the implicit complementarity problem in infinite dimensional spaces.

Let $\left\langle E, E^{*}\right\rangle$ be a dual system of locally convex spaces and let $K \subset E$ be a convex cone.

We denote by $K^{*}$ the dual cone of $K$, that is, $K^{*}=\left\{u \in E^{*} \mid\langle x, u\rangle \geq 0 ; \forall x \in K\right\}$ and if $f: K \rightarrow E^{*}, g: K \rightarrow E$ are given, then the implicit complementarity problem with respect to $f$ and $g$ is,

(I.C.P.) $\| \begin{aligned} & \text { find } x_{0} \in E \text { such that } \\ & g\left(x_{0}\right) \in K, f\left(x_{0}\right) \in K^{*} \text { and }\left\langle g\left(x_{0}\right), f\left(x_{0}\right)\right\rangle=0 .\end{aligned}$
If $g(x)=x ; \forall x \in K$ we have the complementarity problem (C.P.) (or the explicit complementarity problem (E.C.P.)).

Received 31 January 1985.

Copyright Clearance Centre, Inc. Serial-fee code: 0004-9727/85 $\$ A 2.00+0.00$. 
The extensive literature on complementarity problems is motivated by interesting applications in areas as : Optimization Theory, Structural Mechanics, Lubrication Theory, Elasticity Theory, Economical Equilibrium Theory, Equilibrium Theory on Networks, Mathematical Economics Variational Calculus and stochastic Optimal control.

The complementarity problems, till now, have been intensively studied in finite dimensional spaces, but not much in infinite dimensional spaces.

We can find the principal results on complementarity problems in infinite dimensional spaces in, [1], [2], [9], [15], [17-18], [19-21], $[22],[25],[28],[31]$.

The implicit complementarity problem was imposed by some special problems in stochastic optimal control and it was considered by Bensoussan, Lions, Dolcetta, Mosco and Pang in [3-7], [13], [16], [27], $[29-30]$.

It is well known that the theory of explicit complementarity problem is strongly supported by the fixed-point theory.

Recently, in our papers [20-21] we proved, that the theory of coincidence equations on convex cones gives an unified study of the explicit and implicit complementarity problem.

Now, in this paper we consider the implicit complementarity problem in Hilbert spaces and we prove that a generalization of Banach's contraction theorem to coincidences, implies an interesting existence theorem for implicit complementarity problems.

\section{2.}

Let $(E,\|\|)$ be a Banach space and consider, $D \subset E$ a closed subset.

We say that $G: D \rightarrow E$ is a proper mapping if the inverse image of any compact subset of $G(D)$ under $G$ is compact.

The following coincidence theorem is fundamental for our principal result.

THEOREM. [Coincidence]

Let (E, $\|)$ be a Banach space and consider $D \subset E$ a closed subset.

Assume that the continuous moppings $F, G: D \rightarrow E$ satisfy the following assumptions: 
1) $G$ is proper,

2) $F(D) \subset G(D)$,

3) there exists a constant $0<\rho<1$ such that

$$
\|F(x)-F(y)\| \leq \rho\|G(x)-G(y)\|, \forall x, y \in D .
$$

Then, there exists $x_{*} \in D$ such that $F\left(x_{*}\right)=G\left(x_{*}\right)$.

Moreover, if $F$ or $G$ is one to one then the coincidence point $x_{*}$ is unique.

Proof. From (2) there exists a sequence $\left\{x_{n}\right\}_{n \in N}$ defined by $G\left(x_{n+1}\right)=F\left(x_{n}\right) ; \forall n \in N$, where $x_{0}$ is an arbitrary point of $D$.

By recurrence we have,

$$
\left\|G\left(x_{n}\right)-G\left(x_{n+1}\right)\right\|=\left\|F\left(x_{n-1}\right)-F\left(x_{n}\right)\right\| \leq \rho^{n}\left\|G\left(x_{0}\right)-G\left(x_{1}\right)\right\|
$$

$\forall n \in N$, which implies,

$$
\left\|G\left(x_{n}\right)-G\left(x_{n+p}\right)\right\| \leq \frac{\rho}{1-\rho}\left\|G\left(x_{0}\right)-G\left(x_{1}\right)\right\| ; \forall n, p=1,2 \ldots
$$

and, hence, $\left\{G\left(x_{n}\right)\right\}_{n \in N}$ is a cauchy sequence in $E$.

Since $E$ is complete $\left\{G\left(x_{n}\right)\right\}_{n \in N}$ is convergent and because $G$ is a proper mapping the sequence $\left\{x_{n}\right\}_{n \in N}$ has a convergent subsequence

$$
\begin{aligned}
& \left\{x_{\left.n_{k}\right\}} \cdot \dot{N_{k}}\right. \\
& \quad \text { If } x_{*}=\lim _{k \rightarrow \infty} x_{n_{k}} \text {, then from continuity and the definition of }
\end{aligned}
$$
$\left\{x_{n}\right\}_{n \in N}$ we have, $F\left(x_{*}\right)=G\left(x_{\star}\right)$.

If $x_{*}$ and $x_{* *}$ are two elements of $D$, such that, $F\left(x_{*}\right)=G\left(x_{*}\right)$ and $F\left(x_{* *}\right)=G\left(x_{* *}\right)$ then from the following relations,

$$
\begin{aligned}
& \left\|F\left(x_{*}\right)-F\left(x_{* *}\right)\right\| \leq \rho\left\|G\left(x_{*}\right)-G\left(x_{* *}\right)\right\|=\rho\left\|F\left(x_{*}\right)-F\left(x_{* *}\right)\right\|, \\
& \left\|G\left(x_{*}\right)-G\left(x_{* *}\right)\right\|=\left\|F\left(x_{*}\right)-F\left(x_{*}\right)\right\| \leq \rho\left\|G\left(x_{*}\right)-G\left(x_{* *}\right)\right\|,
\end{aligned}
$$

the assumption that $F$ or $G$ is one to one implies that $x_{*}=x_{* *} / /$

REMARK. A very important class of proper mappings for practical problems is the class of the form, $G=L+\mathbb{G}$, where $L$ is a linear Fredholm operator and $\mathbb{Q}$ is a completely continuous nonilinear operator 
in the case where $E$ is a Hilbert space [8].

Consider now, $(H,<,>)$ a Hilbert space and $K \subset H$ a closed convex cone.

If $f, g: K \rightarrow H$ are given, consider again the implicit complementarity problem,

\begin{tabular}{l|l} 
(I.C.P. $)$ & find $x_{0} \in H$ such that, \\
$g\left(x_{0}\right) \in K, f\left(x_{0}\right) \in K^{*}$ and $\left\langle g\left(x_{0}\right), f\left(x_{0}\right)\right\rangle=0$.
\end{tabular}

DEFINITIONS. 1) we say that $f$ is k-Lipschitz with respect to $g$ if,

$$
\|f(x)-j(y)\| \leq k\|g(x)-g(y)\| ; \forall x, y \in K ;\left(k \in \mathbb{R}_{f}\{0\}\right) .
$$

2) The mapping $f$ is called c-strongly monotone

with respect to $g$ if,

$\langle f(x)-f(y), g(x)-g(y)\rangle \geq c\|g(x)-g(y)\|^{2} ; \forall x, y \in K$; where

$c \in \mathbb{R}_{+} \backslash\{0\}$.

THEOREM. 1. Let $(H,<,>)$ be a Hilbert space and $K \subset H$ a closed convex cone. If $f, g: K \rightarrow H$ satisfy the following assumptions:

1) $f$ is k-Lipschitz with respect to $g$,

2) $f$ is c-strongly monotone with respect to $g$,

3) $g$ is a continuous proper mapping and $g(K)=K$,

4) $k^{2}<2 c$,

then there is a solution $y_{0} \in K$ of problem (I.C.P.).

Moreover, if $g$ is one to one on $K$, then the solution $y_{0}$ is mique.

Proof. Since $K$ is a closed, convex subset in $H$ then for any $y \in K$ there is an unique element $\phi(y) \in K$ such that,

$$
\|\phi(y)-g(y)+f(y)\| \leq\|z-g(y)+f(y)\| ; \forall z \in K .
$$

Consider the function $\Psi:[0,1] \rightarrow \boldsymbol{R}_{+}$defined by, $\Psi(\lambda)=\frac{z}{2}\|g(y)-f(y)-(1-\lambda) \phi(y)-\lambda z\|^{2}$, where $z$ is an element in $K$.

obviously, $\Psi$ is a $C^{7}$-function and we have,

$$
\psi^{\prime}(\lambda)=\langle g(y)-f(y)-(1-\lambda \phi(y)-\lambda z, \phi(y)-z\rangle .
$$

Since $\phi(y)$ is the unique element satisfying (1) we have,

(2) :

$$
\Psi^{\prime}(0) \geq 0
$$

and considering the function $\Psi$ for any $z \in K$, from (2) we obtain, 
(3) : $\quad\langle g(y)-j(y)-\phi(y), \phi(y)-z>\geq 0: \forall z \epsilon K$.

Consider now, $y_{1}, y_{2} \in K$, so that $y_{1} \neq y_{2}$ and substituting in (3), $y$ by $y_{1}$ and $z$ by $\phi\left(y_{2}\right)$ we have,

(4) : $\quad\left\langle g\left(y_{1}\right)-f\left(y_{1}\right)-\phi\left(y_{1}\right), \phi\left(y_{1}\right)-\phi\left(y_{2}\right)>\geq 0\right.$

and also, substituting $y=y_{2}$ and $z=\phi\left(y_{1}\right)$, we obtain,

(5) : $\quad\left\langle g\left(y_{2}\right)-f\left(y_{2}\right)-\phi\left(y_{2}\right), \phi\left(y_{2}\right)-\phi\left(y_{1}\right)>\geq 0\right.$.

By addition from (4) and (5) changing the signs in (5) we get,

$$
\begin{aligned}
\left\langle g\left(y_{1}\right)-j\left(y_{1}\right)-y\left(y_{2}\right)+f\left(y_{2}\right), \phi\left(y_{1}\right)-\phi\left(y_{2}\right)\right\rangle \geq \\
\geq\left\langle\phi\left(y_{1}\right)-\phi\left(y_{2}\right), \phi\left(y_{1}\right)-\phi\left(y_{2}\right)\right\rangle=\left\|\phi\left(y_{1}\right)-\phi\left(y_{2}\right)\right\|
\end{aligned}
$$

which implies,

$$
\begin{gathered}
\left\|\phi\left(y_{1}\right)-\phi\left(y_{2}\right)\right\|^{2} \leq k g\left(y_{1}\right)-f\left(y_{1}\right)-g\left(y_{2}\right)+f\left(y_{2}\right), \phi\left(y_{1}\right)-\phi\left(y_{2}\right)>\mid \leq \\
\leq\left\|g\left(y_{1}\right)-f\left(y_{1}\right)-y\left(y_{2}\right)+f\left(y_{2}\right)\right\| .\left\|\phi\left(y_{1}\right)-\phi\left(y_{2}\right)\right\|
\end{gathered}
$$

and finally,

(6) : $\left\|\phi\left(y_{1}\right)-\phi\left(y_{2}\right)\right\|^{2} \leq\left\|f\left(y_{1}\right)-f\left(y_{2}\right)-g\left(y_{1}\right)+g\left(y_{2}\right)\right\|^{2}$.

From assumptions 1), 2) and formula (6) we obtain,

$$
\begin{gathered}
\left\|\phi\left(y_{1}\right)-\phi\left(y_{2}\right)\right\|^{2} \leq\left\|f\left(y_{1}\right)-f\left(y_{2}\right)\right\|^{2}+\left\|g\left(y_{1}\right)-g\left(y_{2}\right)\right\|^{2}- \\
-2\left\langle f\left(y_{1}\right)-f\left(y_{2}\right), g\left(y_{1}\right)-g\left(y_{2}\right)>\leq k^{2}\left\|g\left(y_{1}\right)-g\left(y_{2}\right)\right\|^{2}\right. \\
+\left\|g\left(y_{1}\right)-g\left(y_{2}\right)\right\|^{2}-2 c\left\|g\left(y_{1}\right)-g\left(y_{2}\right)\right\|^{2} .
\end{gathered}
$$

Hence we have the formula,

(7) : $\quad\left\|\phi\left(y_{1}\right)-\phi\left(y_{2}\right)\right\|^{2} \leq\left[k^{2}+1-2 c\right]\left\|g\left(y_{1}\right)-g\left(y_{2}\right)\right\|^{2}$.

Observe now that we can suppose

$$
\text { (8) : } \quad k^{2}<2 c<k^{2}+1
$$

Indeed, if $2 c \geq k^{2}+1$, we can change $c$ to $c_{1}>0$ in definition 2), such that $k^{2}<2 c_{1}<k^{2}+1$, since if $f$ is c-strongly monotone, it is also $c_{1}$-strongly monotone with respect to $g$.

Hence, assuming formula (8), we put $\beta^{2}=k^{2}+1-2 c$ and we obtain that $\phi$ is a $\beta$-contraction with respect to $g$ (since $0<\beta<1$ ). Also, $\phi$ is a continuous mapping (since $g$ is a continuous 
mapping) and we can use the coincidence theorem for $\phi$ and $g$. Thus there is an element $y_{0} \in K$ such that $\phi\left(y_{0}\right)=g\left(y_{0}\right)$.

If we now put $y=y_{0}$ in formula (3) we get,

(9) :

$$
\left\langle f\left(y_{0}\right), z-g\left(y_{0}\right)\right\rangle \geq 0 ; \forall z \in K
$$

which implies (since $0 \in K$ ).

$$
\left\langle f\left(y_{0}\right), g\left(y_{0}\right)\right\rangle \leq 0 \text {. }
$$

Since $K$ is a cone, $2 g\left(y_{0}\right) \in K$ and if we put $z=2 g\left(y_{0}\right)$ in

(9) we obtain,

$$
\left\langle f\left(y_{0}\right), g\left(y_{0}\right)\right\rangle \geq 0
$$

and hence, $\left\langle f\left(y_{0}\right), g\left(y_{0}\right)\right\rangle=0$.

To finish the first part of the proof, we must prove that $f\left(y_{0}\right) \in K^{*}$.

Indeed, let $x \in K$ be an arbitrary element and since $g\left(y_{0}\right) \in K$, we have $x+g\left(y_{0}\right) \in K$ and substituting $z=x+g\left(y_{0}\right)$ in formula (9), we obtain $\left\langle f\left(y_{0}\right), x\right\rangle \geq 0 ; \forall x \in K$, that is, $f\left(y_{0}\right) \in K^{*}$.

Assume now that $g$ is one to one on $K$ and consider two solutions $y_{0}$ and $y_{*}$ of problem (I.C.P.).

The definition of problem (I.C.P.) and assumption 2) imply,

$$
\begin{gathered}
0 \geq\left\langle f\left(y_{0}\right), g\left(y_{0}\right)\right\rangle-\left\langle f\left(y_{*}\right), g\left(y_{0}\right)\right\rangle-\left\langle f\left(y_{0}\right), g\left(y_{*}\right)\right\rangle+\left\langle f\left(y_{*}\right), g\left(y_{*}\right)\right\rangle= \\
=\left\langle f\left(y_{0}\right)-f\left(y_{*}\right), g\left(y_{0}\right)-g\left(y_{*}\right)\right\rangle \geq c\left\|g\left(y_{0}\right)-g\left(y_{1}\right)\right\|^{2}
\end{gathered}
$$

and hence it is necessary to have $y_{0}=y_{*} / /$

COROLLARY 1.Let $(H,<,>)$ be a Hilbert space and $K \subset H$ a closed, convex cone. If $f, g: K \rightarrow H$ satisfy the following assumptions:

1) $f$ is k-Lipschitz,

2) $f$ is a-strongly monotone,

3) $k^{2}<2 c$,

then the explicit complementarity problem (E.C.P.) associated with $f$ has a unique solution.

We say that $g: K \rightarrow H$ is expansive if, there exists $\rho \geq 1$ such that,

$$
\rho\|x-y\| \leq\|g(x)-g(y)\| ; \forall x, y \in K \text {. }
$$


COROLLARY 2. Let $(H,<,>)$ be a Hilbert space and $K \subset H$ a closed convex cone. If $f, g: K \rightarrow H$ satisfy the following assumptions:

1) $g$ is a continuous, proper expansion such that $g(K)=K$,

2) $f$ is a-strongly monotone with respect to $g$ and k-Lipschitz,

3) $k^{2}<2 c$,

then there exists a unique solution of problem (I.C.P.).

Proof. It is sufficient to observe that

$$
\|f(x)-f(y)\| \leq \frac{k}{\rho}\|g(x)-g(y)\| \leq k\|g(x)-g(y)\| ; \forall x, y k . / /
$$

An interesting case for applications is the case of accretive moppings, introduced by Kato [23] and Browder [11] and intensively studied by Browder [10-12], Crandal and Pazy [14], Minty [26], Webb [33] , Schöneberg [32], etc.

The study of accretive mappings is motivated by its applications to nonlinear functional equations and to evolution equations.

If $(E,\|\|)$ is a Banach space, $D \subset E$ a subset and $h: D \rightarrow E$, then $h$ is said to be accretive, if and only if, $\|x-y\| \leq\|(x-y)+\lambda(h(x)-h(y))\|$, for all $x, y \in D$ and $\lambda \geq 0$.

Also, $U: D \rightarrow E$ is said to be pseudo-contractive, if and only if, for all $x, y \in D$ and all $\lambda>0$ we have,

$$
\|x-y\| \leq\|(1+\lambda)(x-y)-(U(x)-U(y))\| \text {. }
$$

An interesting study of pseudo-contractive mappings is[24].

A known result of Kato and Browder [11] is that if $g=I d-U$, where $U: E \rightarrow E$, then $U$ is pseudo-contrative if and only if $g$ is accretive.

COROLLARY 3. Let $(H,<,>)$ be a Hilbert space and $K \subset H$ a closed convex convex cone. If $f, g: K \rightarrow H$ satisfy the following assumptions:

1) $g$ is a continuous, proper mopping such that, $G(K)=K$,

2) $g-p I$ is accretive for some $p>0$ on $K$,

3) $f$ is a-strongly monotone with respect to $g$ and $k$-Lipschitz,

4) $k^{2}<2 c \rho^{2}$,

then problem (I.C.P.) has a solution $y_{0}$.

Moreover, if $\rho \geq 1$, then the solution $y_{O}$ is unique. 
Proof. Indeed, in this case we have,

$$
\|f(x)-f(y)\| \leq \frac{k}{\rho}\|g(x)-g(y)\| ; \forall x, y \in K,
$$

since assumption 2) implies

$$
\begin{aligned}
\|x-y\| \leq \| & (x-y)+\rho^{-1}(g(x)-\rho x-g(y)+\rho y \|= \\
& \left.=\rho^{-1} \| g(x)-g(y)\right) \|,
\end{aligned}
$$

and we have the corollary using theorem 1 .

\section{References.}

[1] Allen G., "Variational inequalities, complementarity problems and duality theorems", J. Hath. Anal. and App Z. 58, (1977), 1-10.

[2] Bazaraa M.S., Goode J.J. and Nashed M.Z., "A nonlinear complementarity problem in mathematical programming in Banach space", Proc. Amer. Math. Soc. Vol. 35, nol, (1972), 165-170.

[3] Bensoussan A., Gourset M. and Lions J.L., "Contrôle impulsionnel et inéquations quasi-variationnelles stationaires", C.R.Acad. Sciences Paris, 276, (1973), A. 1279-1284.

[4] Bensoussan A. and Lions J.L., "Nouvelle formulation des problèmes de contróle impulsionnel et applications", Acad. Sciences Paris. 276, (1973), 1189-1192.

[5] Bensoussan A. and Lions J.L., "Problemes de temps d'arrêt optimal et inéquations variationnelles paraboliques".Applicable Analysis, (1973), 267-294.

[6] Bensoussan A., and Lions J.L., "Nouvelles methodes en controle impulsionnel". Applied Math. and Optimization, no1, (1974), 289-312.

[7] Bensoussan A., "Variational inequalities and optimal stopping time problems".In : D.L. Russel ed: "Calculus of variations and control theory", Academic Press, (1976), 219-244.

[8] Berger M.S. and Schechter M., "On the solvability of semilinear gradient operator equations". Advonces in Math. Vol. 25, (1977), 97-132.

[9] Borwein J.M., "Generalized linear complementariẗy problems treated without fixed-point theory". J. Opt. Theory and Appl. Vol.43 $n^{\circ} 3,(1984), 343-356$. 
[10] Browder F.E., "Nonlinear accretive operators", Bulz. Amer. Math. Soc. 73 , (1967), 470-476.

[11] Browder F.E., "Nonlinear mappings of nonexpansive and accretive type in Banach spaces", Bull. Amer. Math. Soc. 73, (1967), 875-882 .

[12] Browder F.E., "Nonlinear monotone and accretive operators in Banach spaces", Proc. Nat. Acad. Sci. U.S.A. 61, (1968), 388- 393.

[13] Chan D. and Pang J.S., "The generalized quasivariational inequality problem", Math. of Operations Research, Vol. 7, n०2, (1982), 211-222.

[14] Crandal M.G. and Pazy A., "On the range of accretive operators". Israel J. of Math. Vol. 27, nO3-4, (1977) 235-246.

[15] Dash A.T. and Nanda S., "A complementarity problem in mathematical programming in Banach Space", J. Math. AnaZ. and App Z. 98, (1984), 328-331.

[16] Dolcetta I.C. and Mosco V., "Implicit complementarity problems and quasi-variational inequalities",In. R.w. Cottle, F.Giannessi and J.L. Lions eds : "Variational inequalities and complementarity problems", Theory and App l. John Wiley \& Sons, (1980), 75-87.

[17] Fujimoto T., "Nonlinear complementarity problems in a function space", Siam J. Control and Optimization Vol. 18, (1980), 621-623.

[18] Fujimoto T., "An extension of Tarski's fixed point theorem and its applications to isotone complementarity problems", Math. Progromming 28, (1984), 116-118.

[19] Isac G., "Nonlinear complementarity problem and Galerkin method", J. Math. Anal. and Appl. 108 (1985), 563-574.

[20] Isac G., "Complementarity problem via nonlinear eigenvalues problems and coincidence equations on cones", Publ. Math. Universite Limoges, (1984).

[21] Isac G., "Complementarity problem and coincidence equations on convex cones", Preprint (1984).

[22] Karamardian S., "Generalized complementarity problem", J. Optimiz. Theory and App 2. 8, (1971), 161-168. 
[23] Kato T., "Nonlinear semigroups and evolution equations", J. Math. Soc. Japan, (1967), 508-520.

[24] Kirk W.A. and schöneberg R., "Some results an pseudo-contractive mappings", Pacific J. of Math. Vol. 71, nO1, (1977), 89-100.

[25] Luna G., "A remark on the complementarity problem", Proc.Amer. Math. Soc. 48, nol, (1975), 132-134.

[26] Minty G.H., "Monotone (nonlinear) operators in a Hilbert space", Duke Math. J. 29, (1962), 341-346.

[27] Mosco V., "On some non-linear quasi-variational inequalities and implicit complementarity problems in stochastic control theory" In : R.W. Cottle, F. Giannessi and J.L. Lions eds, "Variational inequalities and complementarity problems theory and Applications", John Wiley \& Sons, (1980), 271-283.

[28] Nanda S. and Nanda s., "A nonlinear complementarity problem in mathematical programming in Hilbert space", Bull. Aust. Math. Soc. 20, (1979), 233-236.

[29] Pang J.S., "The implicit complementarity problem", In : O.L. Mangasarian, R.R. Meyer and S.M. Robinson eds., "Nonlinear Programming 4", Academic Press, (1981) 487-518.

[30] Pang J.S., "On the convergence of a basic iterative method for the implicit complementarity problem", J. Optimiz. Theory and App 2. 37, (1982).

[31] Riddell R.C., "Equivalence of nonlinear complementarity problems and least element problems in Banach lattices", Math. of Operations Research. Vol. 6, $\mathrm{n}^{\circ} 3,(1981), 462-474$.

[32] Schöneberg R., "On the domain invariance theorem for accretive mappings", J. London Math. Soc. (2) 24, (1981), 548-554.

[33] Webb G.F., "Nonlinear perturbation of linear operators in Banach spaces", J. Fronct. Anal. 10, (1972), 191-203.

Department de Mathèmatiques,

College Militaire Royal,

st. Jean,

quebec,

Canada JOJ IRO. 